Article

\title{
Cooperation between SS18-SSX1 and miR-214 in Synovial Sarcoma Development and Progression
}

\author{
Miwa Tanaka ${ }^{1}$, Mizuki Homme ${ }^{1}$, Yukari Yamazaki ${ }^{1}$, Keisuke Ae ${ }^{2}$, Seiichi Matsumoto ${ }^{2}$, \\ Subbaya Subramanian ${ }^{3}$ (D) and Takuro Nakamura ${ }^{1, * \mathbb{D}}$ \\ 1 Division of Carcinogenesis, The Cancer Institute, Japanese Foundation for Cancer Research, Tokyo 135-8550, \\ Japan; miwa.tanaka@jfcr.or.jp (M.T.); mizuki.homme@jfcr.or.jp (M.H.); yukari.yamazaki@jfcr.or.jp (Y.Y.) \\ 2 Department of Orthopedic Oncology, The Cancer Institute Hospital, Japanese Foundation for Cancer \\ Research, Tokyo 135-8550, Japan; keisuke.ae@jfcr.or.jp (K.A.); smatsumoto@jfcr.or.jp (S.M.) \\ 3 Department of Surgery, University of Minnesota, Minneapolis, MN 55455, USA; subree@umn.edu \\ * Correspondence: takuro-ind@umin.net; Tel.: +81-3-3570-0462
}

Received: 26 December 2019; Accepted: 22 January 2020; Published: 30 January 2020

\begin{abstract}
SS18-SSX fusion proteins play a central role in synovial sarcoma development, although, the genetic network and mechanisms of synovial sarcomagenesis remain unknown. We established a new ex vivo synovial sarcoma mouse model through retroviral-mediated gene transfer of SS18-SSX1 into mouse embryonic mesenchymal cells followed by subcutaneous transplantation into nude mice. This approach successfully induced subcutaneous tumors in $100 \%$ recipients, showing invasive proliferation of short spindle tumor cells with occasional biphasic appearance. Cytokeratin expression was observed in epithelial components in tumors and expression of TLE1 and BCL2 was also shown. Gene expression profiling indicated SWI/SNF pathway modulation by SS18-SSX1 introduction into mesenchymal cells and Tle1 and Atf 2 upregulation in tumors. These findings indicate that the model exhibits phenotypes typical of human synovial sarcoma. Retroviral tagging of the tumor identified 15 common retroviral integration sites within the Dnm3 locus as the most frequent in 30 mouse synovial sarcomas. miR-199a2 and miR-214 upregulation within the Dnm3 locus was observed. SS18-SSX1 and miR-214 cointroduction accelerated sarcoma onset, indicating that miR-214 is a cooperative oncomiR in synovial sarcomagenesis. miR-214 functions in a cell non-autonomous manner, promoting cytokine gene expression (e.g., Cxcl15/IL8). Our results emphasize the role of $m i R-214$ in tumor development and disease progression.
\end{abstract}

Keywords: synovial sarcoma; SS18-SSX1; mouse model; insertional mutagenesis; miR-214

\section{Introduction}

Synovial sarcoma is a malignant mesenchymal neoplasm characterized by SS18-SSX gene fusions associated with $\mathrm{t}(\mathrm{X} ; 18)$ chromosome translocation, characteristic biphasic morphologies consisting of both mesenchymal and epithelial components, highly invasive growth, and poor prognosis [1-3]. SS18-SSX1/SSX2/SSX4 fusions were observed in most synovial sarcoma [4]. The SS18-SSX fusion proteins show pleiotropic functions that promote oncogenesis [5]. SS18-SSX1 competes with wild type SS18, a component of the SWI/SNF complex, hijacking the complex, inducing aberrant expression of Sox 2 and perturbating the interaction between polycomb and SWI/SNF complexes [6,7]. SS18-SSX1 also interacts with the transcriptional co-repressor TLE1 and recruits it to ATF2, resulting in abnormal repression of ATF2 target genes such as EGR1 [8]. In addition, SWI/SNF disruption by SS18-SSX fusion proteins induces activation of WNT/ $\beta$-catenin signaling [9]. Another study showed that KDM2B and polycomb repressive complex 1 recruits SS18-SSX1 proteins and the SWI/SNF complex to unmethylated CpG islands, leading to aberrant activation of gene expression [10]. Although PTEN, CTNNB1, and APC 
mutations have been reported in synovial sarcoma, the frequency of secondary mutations in this disease is not very high [11]. Moreover, the cell-of-origin of synovial sarcoma remains unclear [1], indicating that the genetic/epigenetic events that cooperate with SS18-SSX should be clarified.

A genetically engineered mouse model of human synovial sarcoma has been previously created by introducing a conditional allele of SS18-SSX2 into the ROSA26 locus, after which the knock-in mice were by mated with Myf5-Cre transgenic mice. As a result, the biphasic synovial sarcoma phenotype was well recapitulated [12], indicating that SS18-SSX2 expression efficiently induces synovial sarcoma in a specific cellular lineage. We have previously generated mouse models of fusion gene-associated sarcoma by introducing EWS-FLI1, ASPSCR1-TFE3, or CIC-DUX4 into mouse embryonic mesenchymal cells (eMCs) [13-15]. In these models, induced tumors can recapitulate phenotypes of human counterparts and are therefore useful as platforms for exploring novel disease mechanisms and testing the effects of new drugs.

In this study, we generated a new mouse model of human synovial sarcoma by using the ex vivo gene manipulation technology and mouse embryonic mesenchymal cells. Moreover, the retrovirus-mediated gene transfer procedure allowed us to perform retroviral tagging and to identify novel cooperative genes for SS18-SSX1 in synovial sarcoma development. We showed that expression of microRNA miR-214 accelerates the onset of synovial sarcoma. Given that overexpression of $m i R-214$ in human synovial sarcoma has been previously reported [16], our present results shed light on the mechanisms of synovial sarcoma development and progression.

\section{Results}

\subsection{Generation and Characterization of the Ex Vivo Model of Synovial Sarcoma}

In previous studies, we generated mouse models of human sarcoma expressing fusion genes such as Ewing sarcoma, alveolar soft part sarcoma, and CIC-DUX4 sarcoma [13-15]. Our work emphasizes the advantages of using mouse eMCs to concentrate a cell-of-origin for fusion gene-associated sarcomas. In this context, we retrovirally introduced human SS18-SSX1 into murine eMCs derived from embryo extremities on dpc 18.5. Transduced eMCs were then subcutaneously injected into nude mice. Recipient mice developed a subcutaneous mass with $100 \%$ penetrance and a mean latency of 26.6 weeks (Figure 1A). These tumors were slow growing and invaded surrounding tissues but without showing distant metastasis.

Histological analysis showed that SS18-SSX1-expressing tumors were composed of a diffuse proliferation of short spindle cells with or without tubulus-like epithelial structures, equivalent to human biphasic or monophasic synovial sarcoma, respectively (Figure 1B). Of the 59 sarcomas, 44 were monophasic and 15 were biphasic. Sarcoma cells showed diffuse infiltration into the surrounding tissues such as skeletal muscles where intra-fascicular invasion was evident (Figure 1C). Expression of FLAG-tagged SS18-SSX1 fusion proteins was confirmed by immunoblotting (Figure 1D), and nuclear accumulation of fusion proteins was observed both in spindle and epithelial elements (Figure 1E). Immunostaining showed expression of cytokeratin (AE1/AE3) not only in epithelial-like cells but also in spindle cells in 19 out of 20 tumors. Enhanced expression of BCL2 and TLE1 was consistently observed in 20 out of 20 tumors with observations in the human counterpart (Figure 1F) [17,18]. These tumors were transplantable into nude mice and Balb/c mice with similar morphological characteristics (Figure 1G), indicating that the tumors were not transient overgrowths of eMCs. Together, these results show that introduction of SS18-SSX1 into eMCs can induce sarcomas which recapitulated the phenotypes of human synovial sarcoma, such as typical biphasic morphologies and expression of synovial sarcoma-specific biomarkers. 
A

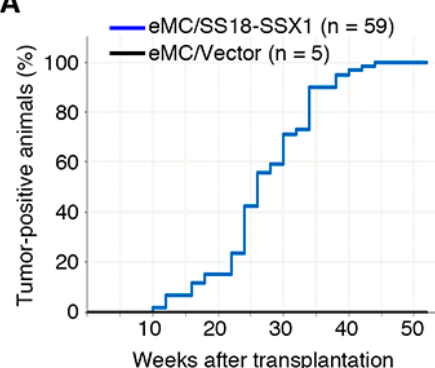

D

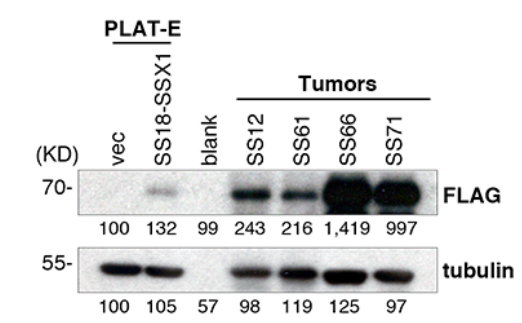

F

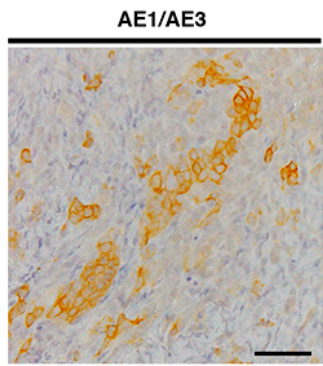

B

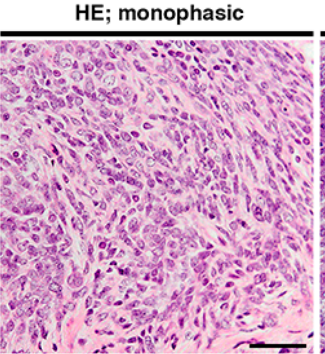

E
FLAG; monophasic

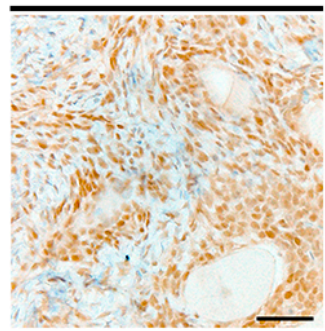

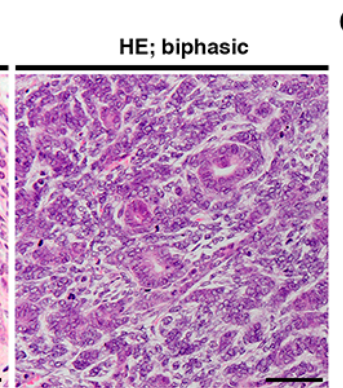

C
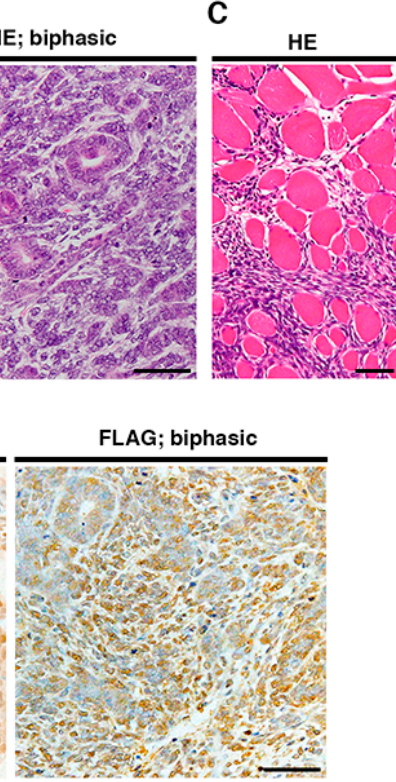

G
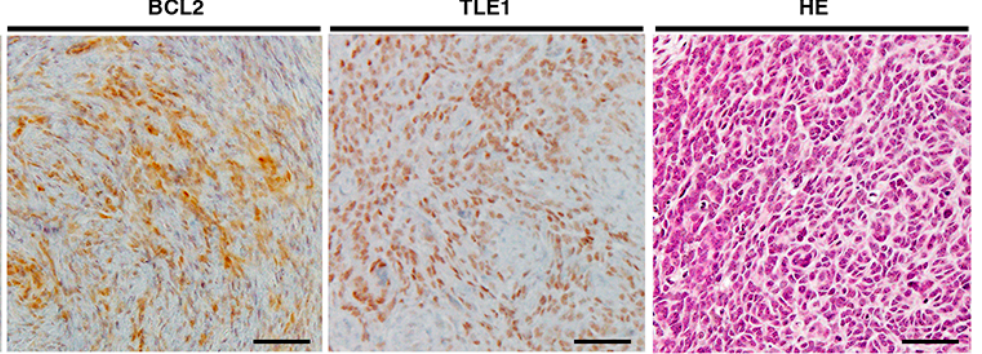

Figure 1. Generation of the mouse synovial sarcoma ex vivo model. (A) Cumulative incidences of synovial sarcoma. (B) Histology of mouse synovial sarcoma showing monophasic (left) and biphasic (right) patterns. (C) Infiltration of sarcoma cells into surrounding muscle tissue. (D) Immunoblotting of FLAG-SS18-SSX1 in tumor tissue. PLAT-E cells transduced with SS18-SSX1 or empty vector are used as positive and negative controls, respectively. (E) Accumulations of SS18-SSX1 in the nucleus of tumor cells are evident in both monophasic and biphasic tumors by immunostaining with anti-FLAG. (F) Immunostaining with anti-AE1/AE3, anti-BCL2, and anti-TLE1. (G) Histology of the secondary transplanted tumor. Scale bars indicate $50 \mu \mathrm{m}$.

\subsection{Gene Expression Profile of Mouse Synovial Sarcoma}

Microarray analyses were carried out to examine the gene expression profile of mouse synovial sarcomas and mouse eMCs with or without introduction of SS18-SSX1 at $48 \mathrm{~h}$ after introduction. Gene set enrichment analysis (GSEA) using gene sets for eMCs showed enrichment of the SNF5 gene set containing genes upregulated in mouse embryonic fibroblasts with Snf5 knockout [19] in the SS18-SSX1-expressing cohort (Figure 2A and Table S1), and the changes were detected $48 \mathrm{~h}$ after SS18-SSX1 introduction. Since disruption of the SNF/SWI complex by SS18-SSX1 was observed in human synovial sarcoma cell lines [7], the present result suggests that affection of the pathway occurred at the early stage of synovial sarcoma development. Representative downstream genes in the SNF5 pathway, Sox2 and ApoD, were significantly upregulated in eMCs expressing SS18-SSX1 and also in mouse synovial sarcomas (Figure 2B). Expression profiles of mouse synovial sarcomas were also analyzed, and enriched genes within Cyclin D1, PTEN, and $\beta$-catenin pathways were also found to be upregulated (Figure $2 \mathrm{C}$ and Table S1). Upregulation of ATF2 and TLE1 is important in the development of human synovial sarcoma and recruitment of TLE1 to the ATF2 binding locus downregulates expression of the tumor suppressor $E G R 1$ [8,20,21]. In accordance with this, expression of Atf2 and Tle1 was increased, while that of Egr1 was suppressed in mouse synovial 
sarcoma (Figure 2D). These changes were not observed in eMCs expressing SYT-SSX1, suggesting that the expression modulation of the Atf2/Tle1/Egr1 axis might be achieved at a later stage. However, Igf1, which is downstream of the Cyclin D1 pathway, was upregulated both in tumors and eMCs expressing SS18-SSX1, which might be important for drug resistance in synovial sarcoma treatment [22]. Overall comparison of differentially expressed genes between SS18-SSX1-expressing eMCs and tumors showed that 3155 and 485 genes were upregulated in synovial sarcoma versus eMCs with an empty vector; fold change > 2.0, and in SS18-SSX1-expressing eMCs versus eMCs with an empty vector; fold change > 2.0, (Figure 2E and Table S2). Among these genes commonly upregulated genes in SS18-SSX1-expressing eMCs and tumors, such as Sox 2 and ApoD, might be downstream targets of the SS18-SSX1 and SWI/SNF axis, and their expression is preserved during oncogenesis, whereas tumor-specific upregulated genes such as Atf2 and Tle1 might require other factors for their expression at the later stage.

A

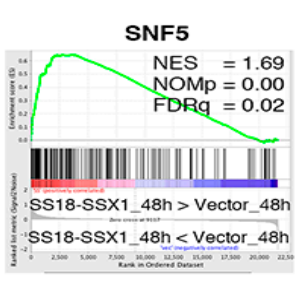

C

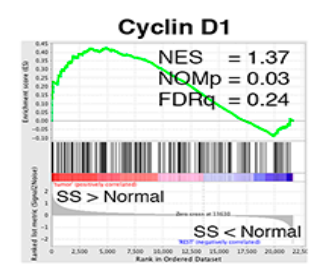

B
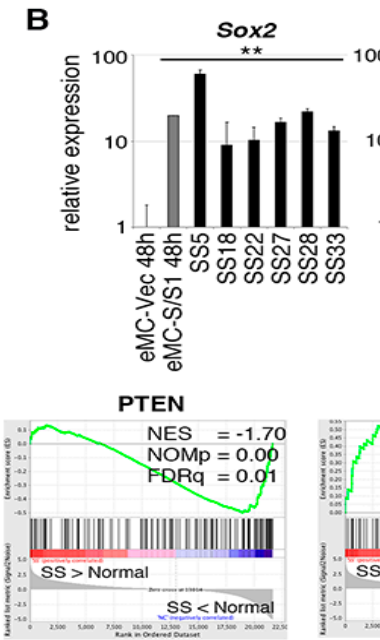

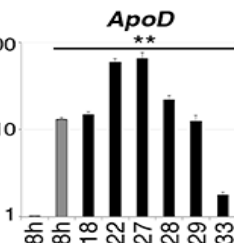

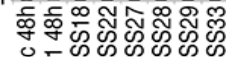

क्ष

$\sum_{0}^{0}$

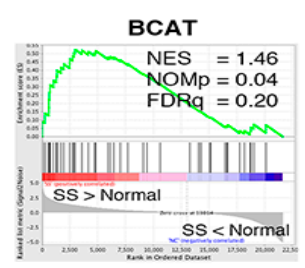

D
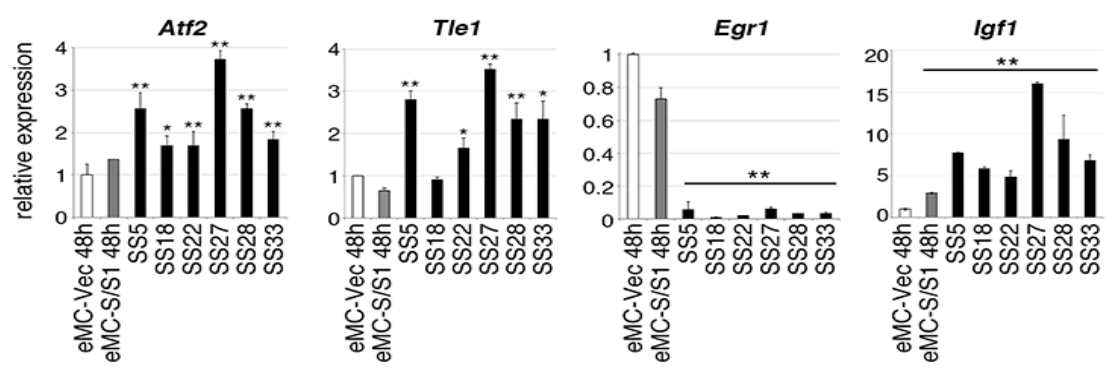

$\mathbf{E}$
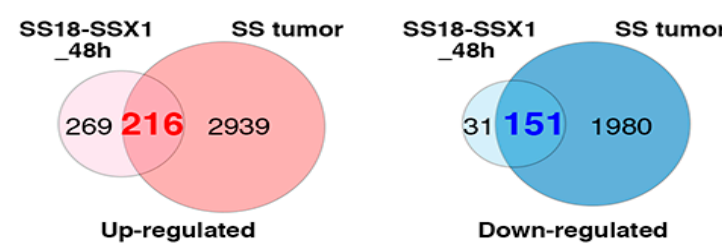

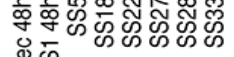

这官

ऐंऐo

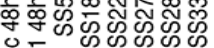

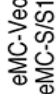

Figure 2. Gene expression profiling of mouse synovial sarcoma and embryonic mesenchymal cells (eMCs) expressing SS18-SSX1. (A) Gene set enrichment analysis (GSEA) of eMCs expressing SS18-SSX1 versus eMCs with an empty vector shows enrichment of the SNF5 pathway. (B) Real-time quantitative RT-PCR for Sox2 and ApoD in eMCs with or without SS18-SSX1, and synovial sarcoma tissues. Expression values were normalized to Gapdh expression. ${ }^{* *} p<0.01$. (C) GSEA of mouse synovial sarcomas versus mouse normal tissue mix shows enrichment of the Cyclin D1, Phosphatase and Tensin Homolog (PTEN), and $\beta$-catenin pathways. (D) RT-qPCR for Atf2, Tle1, Egr1, and Igf1 in eMCs with or without SS18-SSX1, and synovial sarcoma tissues. Expression values were normalized to Gapdh expression. ${ }^{*} p<0.05$ and ${ }^{* *} p<0.01$. (E) Venn diagrams for upregulated and downregulated genes in eMCs expressing SS18-SSX1 (versus eMCs with empty vector) and mouse synovial sarcomas (versus mouse normal tissue mix). Fold changes $>2.0, p<0.05$. 


\subsection{Retroviral Tagging Identifies Candidate Cooperative Genes for SS18-SSX1}

In the present mouse synovial sarcoma model, SS18-SSX1 was introduced using a murine myeloid leukemia virus-based retroviral vector, which enabled aberrations of genes cooperating with SS18-SSX1 via retroviral integrations [23-25]. Southern blotting showed that tumors showed integrations of multiple retroviral copies (Figure S1A). To identify such cooperative genes, 269 retroviral integration sites were isolated and 15 were common in 30 synovial sarcomas (Figure 3A, Tables 1 and S3). The most frequent integration site was the Dnm3 locus on chromosome 1 in which three tumors, SS18, SS19, and SS23, showed retroviral insertions (Figure 3B). These integrations were observed in introns of Dnm3 without upregulating its expression (Figure S1B). There is a Dnm3os long non-coding RNA in intron 14 of Dnm3 with opposite transcriptional direction. In addition, Dnm3os contains two microRNAs, $m i R-199 a 2$ and $m i R-214$ (Figure 3B) [26].

Expression of miR-199a2 and miR-214 were significantly upregulated in synovial sarcomas (SS18, SS19, and SS23) with retroviral integrations, although the upregulation of miR-214 in SS23 was moderate (Figure 3C). It is possible that the retroviral integration in the SS23 locus might be present only in a minor subpopulation of the tumor. Moreover, miR-199a2 upregulation was observed only in one tumor (SS19) with retroviral integrations, suggesting that miR-199a2 might not be a target of the integration as was also indicated by the co-expression experiment (see below). miR-214 expression was also upregulated in human synovial sarcoma as reported previously [16], and its expression was the highest in sarcomas of six types (Figure S2A). Increased Dnm3os expression was also observed both in human and mouse synovial sarcomas compared with Ewing sarcoma, alveolar soft part sarcoma, or rhabdomyosarcoma (Figure S2B), suggesting the importance of the locus in synovial sarcoma development.

A

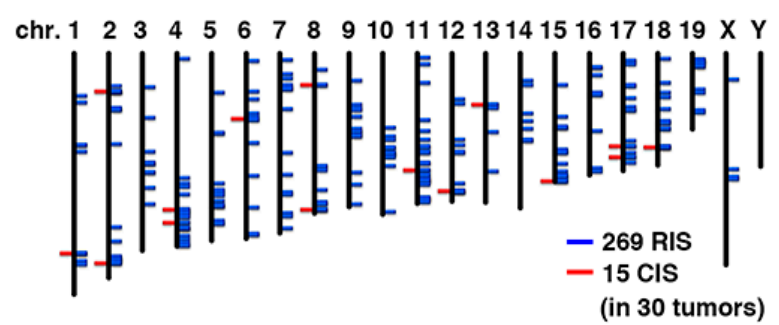

C
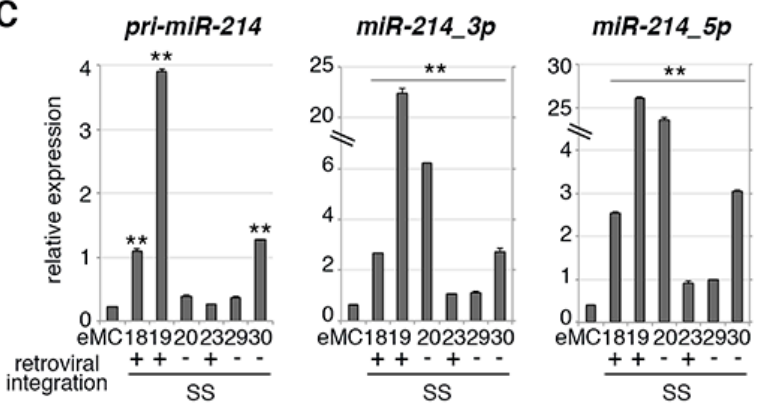

B

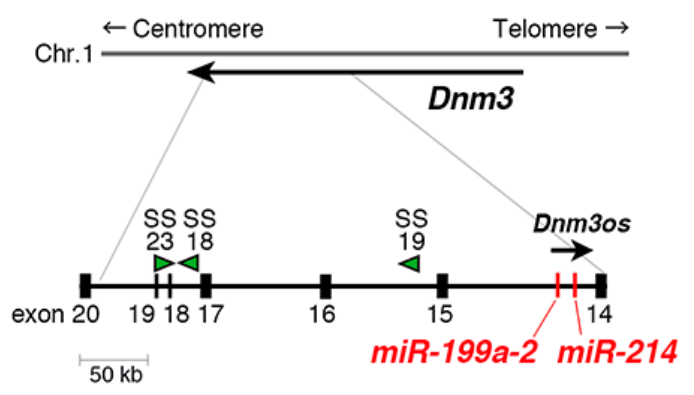

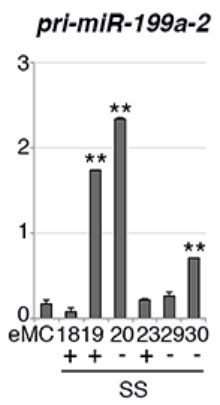
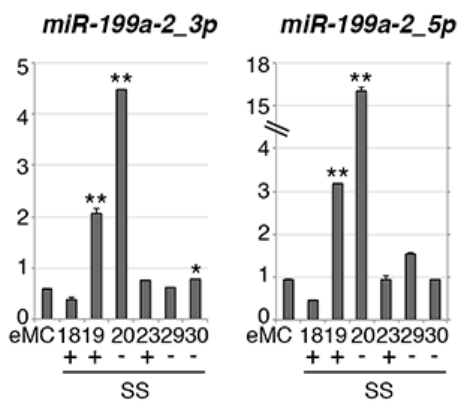

Figure 3. Identification of retroviral integration sites in mouse synovial sarcomas. (A) Distribution of 269 retroviral integration sites in 30 tumors on mouse chromosomes. Common integration sites are indicated with red lines, and single integration sites with blue lines. (B) Schematic of retroviral integrations at the Dnm3 locus. Each integration is marked with arrowheads with the directions also indicated. Genetic positions of Dnm3os, miR-214, and miR-199a-2 are indicated. (C) RT-qPCR for pri-miR-214, miR-214_3p, miR-214_5p, pri-miR-199a-2, miR-199a-2_3p, and miR-199a-2_5p in synovial sarcomas with or without retroviral integrations. Expression values were normalized to snoRNA202 expression. ${ }^{*} p<0.05$ and ${ }^{* *} p<0.01$. 
Table 1. Common retroviral integration sites in murine synovial sarcoma.

\begin{tabular}{ccc}
\hline Chromosome Locus & Loci & No. of Integrations $(\boldsymbol{n}=\mathbf{3 0})$ \\
\hline $1 \mathrm{qH} 2.1$ & Dnm3 & 3 \\
\hline $2 \mathrm{qH} 3$ & Tshz2 & \\
$2 \mathrm{qB}$ & Cstad & \\
$4 \mathrm{qD} 2.2$ & Stk40 & \\
4qD3 & Hspg2 & \\
6qB3 & Creb5 & \\
8qA2 & Fgfr1 & \\
8qE2 & Irf2bp2 & \\
$11 \mathrm{qD}$ & Col1a1 & \\
$12 \mathrm{qF} 1$ & Trmt61a & \\
$13 \mathrm{qA} 4$ & Nedd9 & \\
$15 \mathrm{qF3}$ & Itga5 & \\
$17 \mathrm{qE} 2$ & Ltbp1 & \\
$17 \mathrm{qE} 4$ & $4933433 H 22 R i k$ & \\
$18 \mathrm{qE} 3$ & Zbtb7c & \\
\hline
\end{tabular}

\section{4. miR-214 Cooperates with SS18-SSX1 in Synovial Sarcoma Development}

To examine the cooperativity between SS18-SSX1 and miR-199a2 or miR-214, SS18-SSX1, either microRNA sequences were coexpressed in eMCs and subjected to sarcoma induction experiments (Figure 4A). Coexpression of $m i R-214$ but not miR-199a2 significantly accelerated SS18-SSX1-induced synovial sarcoma development with 14.4 and 29.2 weeks of mean latency, respectively (Figure 4B). Expression of $F g f r 1$ and Nedd9, also located near common integration sites, did not accelerate synovial sarcoma development (Figure S3). miR-214 expression was compared between SS18-SSX1/miR-214 co-transduced tumors and SS18-SSX1 only tumors, and a significant inverse correlation was observed between miR-214 expression and the sarcoma latency (Figure 4C). miR-214-positive sarcomas showed similar morphological characteristics to SS18-SSX1 only sarcomas, namely monophasic or biphasic patterns composed of short spindle tumor cells (Figure 4D), and there was no significant difference in SS18-SSX1 protein expression (Figure 4E). In situ hybridization showed miR-214 expression in both the nucleus and cytoplasm of SS18-SSX1- and miR-214-coexpressing tumor cells (Figure 4F), suggesting that miR-214 mainly functions in tumor cells. Subsequently, we found that expression of $m i R-214$ was correlated with a worse prognosis in human synovial sarcoma cases (Figure 4G). 
A

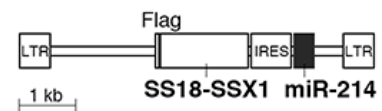

D

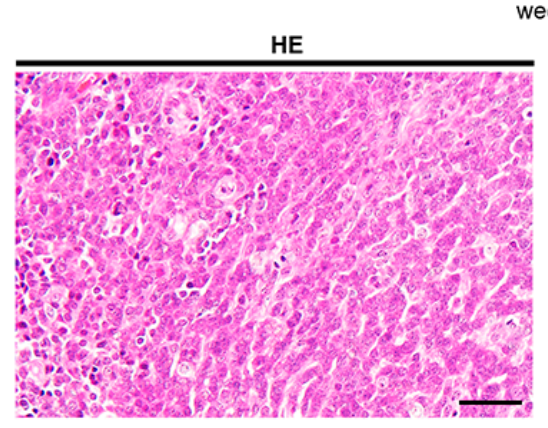

$\mathbf{F}$

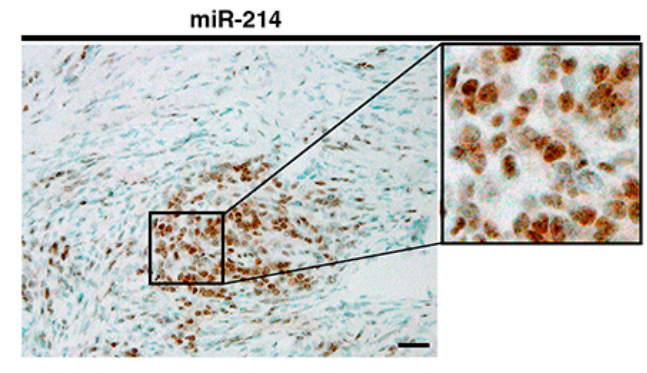

miR-214
B

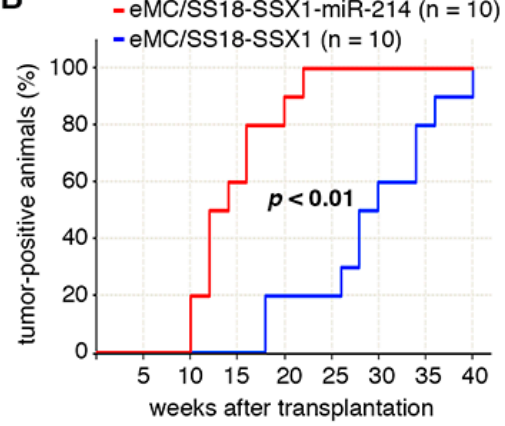

C

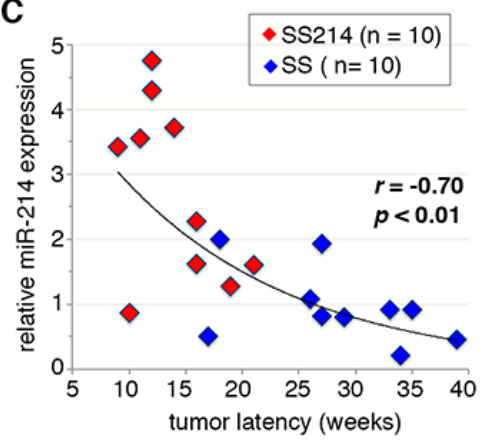

G

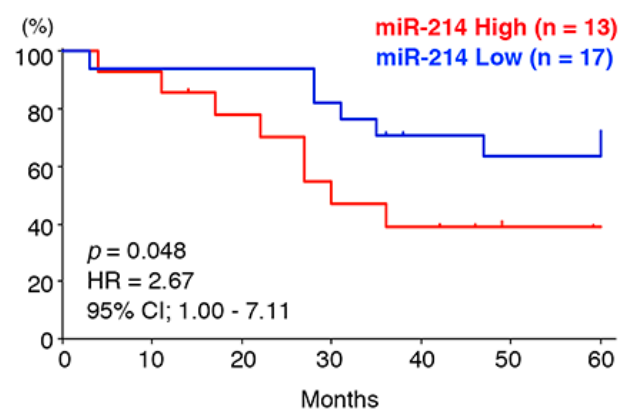

Figure 4. miR-214 cooperates with SS18-SSX1 in synovial sarcoma development. (A) Schematic of pMYs-SS18-SSX1-IRES-miR-214/miR-199a-2. (B) Cumulative incidences of synovial sarcoma with or without miR-214. Significance was examined by log-rank test. (C) Pearson's correlation analysis shows inverse correlation between miR-214 expression and disease latencies. (D) Histology of synovial sarcoma expressing both SS18-SSX1 and miR-214. Scale bar indicates $50 \mu \mathrm{m}$. (E) Immunoblotting of FLAG-SS18-SSX1 in tumor tissue shows no significant difference in expression between sarcomas with and without $m i R-214$. (F) In situ hybridization of $m i R-214$ shows sarcoma cell-specific expression of $m i R-214$. Inset clarifies nuclear and cytoplasmic accumulation of $m i R-214$. Scale bar indicates $50 \mu \mathrm{m}$. (G) Kaplan-Meier survival curves show that increased expression of miR-214 is associated with poor prognosis in human synovial sarcoma cases.

\subsection{Effects of miR-214 Expression on Synovial Sarcoma}

Gene expression profiles were compared between synovial sarcomas expressing SS18-SSX1 $(n=12)$ and SS18-SSX1 with $m i R-214(n=13)$. The principal component analysis and unsupervised clustering showed that both cohorts had distinct expression profiles in both subtypes with a minor exception (Figure 5A,B). We selected 168 genes that were downregulated following introduction of miR-214 in two different synovial sarcoma cell lines, SS40 and SS60, and 53 genes that were potential miR-214 targets were further selected by a database search (miRbase; http:/www.mirbase.org and TargetScan; http://www.targetscan.org/vert_72/). Among these 53 genes, expression of 11 genes (Timp2, Ezh1, Foxo4, Nomo1, Pten, Tpp1, Plagl2, Chfr, Fbxo3, Lats2, and Cbl) that are predicted targets of miR-214 were further analyzed because of their known involvement in cancer (Figures 5C and S4A). RT-qPCR showed downregulation of Timp2, Ezh1, and Pten and immunoblotting showed that the effect of 
$m i R-214$ on these genes was rather limited (Figure 5C,D). Nevertheless, exogenous introduction of miR-214 or Dnm3os into synovial sarcomas and silencing of miR-214 by anti-miR-214 did not induce growth promotion, migration, or invasion (Figure S4B-D), suggesting that miR-214 might not function in a cell intrinsic manner.

A

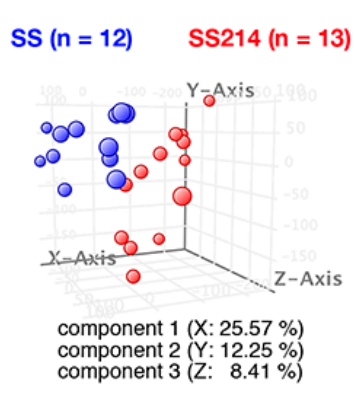

D

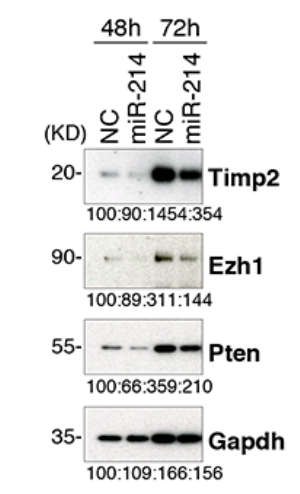

E

Top 5 Diseases and Disorders

\begin{tabular}{lc}
\multicolumn{2}{c}{ Top 5 Diseases and Disorders } \\
\hline name & $p$-value \\
\hline Inflammatory Response & $3.72 \mathrm{E}-03-1.28 \mathrm{E}-09$ \\
Immunological Disease & $3.72 \mathrm{E}-03-5.55 \mathrm{E}-07$ \\
Connective Tissue & $3.72 \mathrm{E}-03-7.20 \mathrm{E}-07$ \\
\multicolumn{1}{c}{ Disorders } & \\
Inflammatory Disease & $3.72 \mathrm{E}-03-7.20 \mathrm{E}-07$ \\
Organismal injury and & $3.72 \mathrm{E}-03-7.20 \mathrm{E}-07$ \\
\multicolumn{2}{c}{ Abnormalities } \\
\hline
\end{tabular}

B

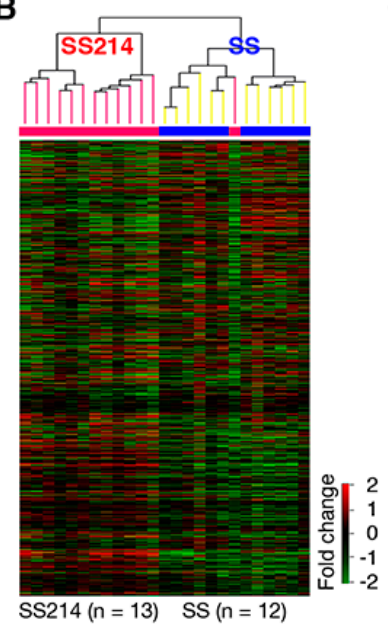

F

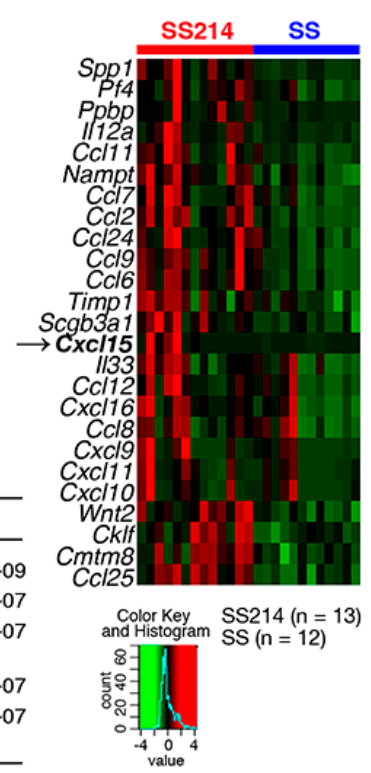

C
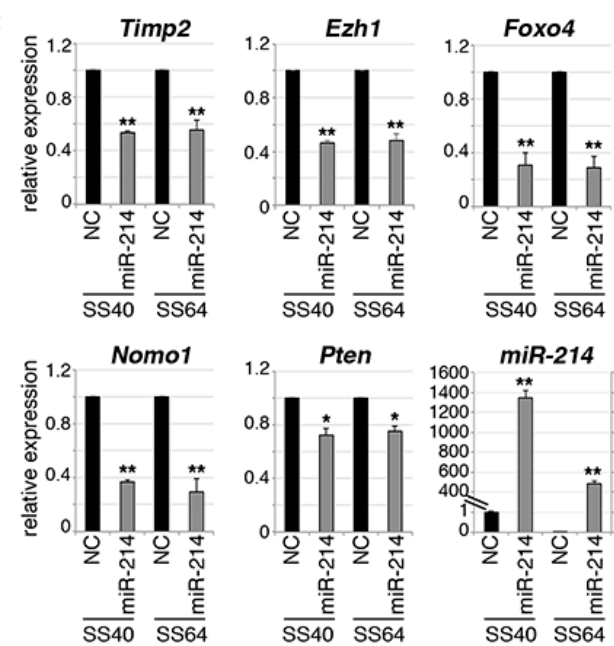

G

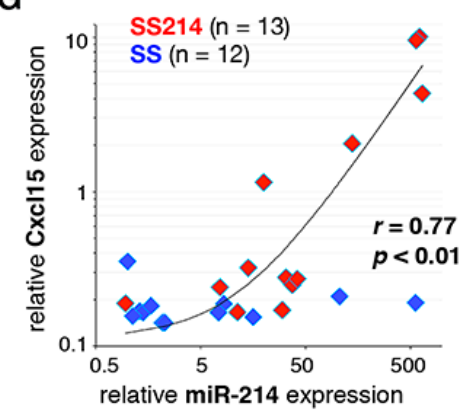

H

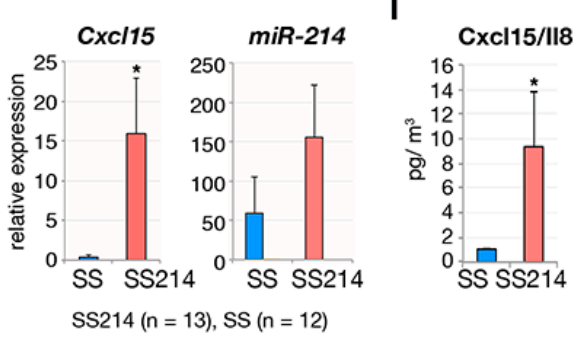

Figure 5. miR-214 expression modulates gene expression in mouse synovial sarcoma. (A) Principal component analysis and (B) Hierarchical clustering of gene expression profiles of synovial sarcoma with and without $m i R-214$ overexpression. (C) RT-qPCR for candidate miR-214 target genes, Timp2, Ezh1, Foxo4, Nomo1, and Pten in synovial sarcoma cells with or without miR-214. ${ }^{*} p<0.05,{ }^{* *} p<0.01$. (D) Immunoblotting for Timp2, Ezh1, and PTEN in the SS40 mouse synovial sarcoma cell with or without miR-214 introduction. (E) Ingenuity Pathway Analysis (IPA) of signaling pathways for 272 upregulated genes in synovial sarcoma expressing miR-214. The key diseases and disorders are listed according to their ranking scores. (F) A heatmap of top 25 cytokine genes upregulated in synovial sarcoma with miR-214 expression. The arrow indicates Cxcl15. (G) Pearson's correlation analysis shows positive correlation between miR-214 and Cxcl15 expression in mouse synovial sarcomas. (H) Quantitative RT-PCR showed Cxcl15 upregulation in miR214-positive synovial sarcomas. Expression of miR-214 is shown in the right panel. Cxcl15 expression relative to Gapdh and miR-214 relative to sno202 are shown. (I) IL-8 protein expression is compared between mouse synovial sarcomas with and without miR-214. 
Ingenuity pathway analysis (IPA) of differentially expressed genes between synovial sarcomas with and without $m i R-214$ expression showed an enrichment for inflammatory response genes (Figure 5E and Table S4). In addition, a series of cytokine and chemokine genes were upregulated in synovial sarcomas expressing miR-214 (Figure 5F and Table 2). Cytokine/chemokine expression was compared between human synovial sarcoma and other sarcomas such as osteosarcoma, Ewing sarcoma, alveolar soft part sarcoma, and rhabdomyosarcoma using publicly available databases (Table 3). The mouse Cxcl15/Il8, a counterpart of human CXCL8/IL8, was upregulated in both human synovial sarcoma and mouse synovial sarcoma with miR-214 expression. Microarray analyses of mRNA and microRNA expression in mouse synovial sarcoma showed a strong positive correlation $(\mathrm{r}=0.77, p<0.01)$ between $\mathrm{Cxcl15}$ and miR-214 expression (Figure 5G). Cxcl15 transcript and protein levels in miR-214-positive synovial sarcomas was confirmed by RT-qPCR and ELISA, respectively (Figure 5H,I). Cxcl15/IL-8 induces migration of macrophages and granulocytes. However, the number of CD163-positive macrophages and naphthol AS-D chloroacetate esterase-positive granulocytes were not different between synovial sarcoma with and without $m i R-214$ expression (Figure S4E).

Table 2. Upregulated cytokine genes in mouse synovial sarcoma *.

\begin{tabular}{ccc}
\hline Genes & Fold Change & Products \\
\hline Cxcl15 & 12.34 & chemokine (C-X-C motif) ligand 15 \\
Ppbp & 5.80 & pro-platelet basic protein \\
Spp1 & 4.31 & secreted phosphoprotein 1 \\
C cl6 & 3.63 & chemokine (C-C motif) ligand 6 \\
C cl9 & 3.57 & chemokine (C-C motif) ligand 9 \\
C cl11 & 2.48 & chemokine (C-C motif) ligand 11 \\
Cxcl9 & 2.21 & chemokine (C-X-C motif) ligand 9 \\
C cl2 & 2.16 & chemokine (C-C motif) ligand 2 \\
Pf4 & 2.13 & platelet factor 4 \\
Ccl7 & 1.88 & chemokine (C-C motif) ligand 7
\end{tabular}

* Upregulated genes in SS18-SSX1- and miR-214-coexpressing tumors (versus SS18-SSX1 only tumors). Cxc115 that is upregulated both in mouse and human synovial sarcoma is indicated in bold.

Table 3. Upregulated cytokine genes in human synovial sarcoma *.

\begin{tabular}{ccl}
\hline Genes & Fold Change & \\
\hline BMP7 & 6.79 & Products \\
BMP5 & 4.48 & bone morphogenetic protein 7 \\
WNT5A & 4.35 & wingless-type MMTV integration site family, member 5A \\
CXCL12 & 3.35 & chemokine (C-X-C motif) ligand 12 \\
TNFRSF19 & 2.75 & tumor necrosis factor receptor superfamily, member 19 \\
BMP2 & 2.46 & bone morphogenetic protein 2 \\
TGFB2 & 2.14 & transforming growth factor beta 2 \\
CXCL8 & 1.86 & chemokine (C-X-C motif) ligand 8 \\
FAM19A1 & 1.63 & family with sequence similarity 19 (chemokine (C-C motif)-like), member A1 \\
CCL28 & 1.60 & chemokine (C-C motif) ligand 28 \\
\hline
\end{tabular}

${ }^{*}$ Upregulated genes in human synovial sarcoma versus other sarcomas (osteosarcoma, Ewing sarcoma, alveolar soft part sarcoma, and rhabdomyosarcoma). CXCL8 that is upregulated both in mouse and human synovial sarcoma is indicated in bold.

\section{Discussion}

Our mouse model of synovial sarcoma showed faithful reproduction of the phenotype of its human counterpart using eMCs as the cell-of-origin. Biphasic morphology consisting of both mesenchymal and epithelial components, a hallmark of synovial sarcoma pathology, was observed in $25 \%$ of all tumors. The incidence of the biphasic subtype was low but cytokeratin-positive tumor cells were frequently observed in the monophasic mesenchymal subtype, indicating that there is transition between the two components. Previous studies showed that myoblast-specific expression of SS18-SSX2 
induced synovial sarcoma phenotypes in mouse [12], suggesting that myogenic progenitors might be one of the cell-of-origin of synovial sarcoma. Although our current model did not contribute to the topological information for the cell-of-origin, our results using embryonic mesenchymal cells suggest an association between some developmental abnormalities and synovial sarcomagenesis.

The micro RNAs miR-214 and miR-199a-2 are embedded as parts of lncRNA Dnm3os within the Dnm3 locus [24]. The locus is well conserved during evolution and common target genes of $m i R-214$ have been reported among human, mouse, and zebrafish [27]. Mesenchyme-specific expression of Dnm3os has been reported and Dnm3os knockout mice show severe abnormalities in musculoskeletal development [26-29]. The importance of $m i R-214$ in mesenchymal tissue and the high level of expression of Dnm3os suggests the presence of $m i R$-214-expressing clusters in embryonic mesenchymal cells. Although upregulation of $m i R-214$ in our mouse model was achieved by insertions of retroviral sequences that act as an enhancer [23], the mechanism of miR-214 and DNM30S upregulation in human synovial sarcoma remains unclear. In addition, $m i R-214$ and $m i R-199 a$ upregulation was observed in several mouse synovial sarcomas without retroviral integrations. Expression of Dnm3os suggests epigenetic regulation of the locus in the developing mesenchymal tissues, and further studies are needed to clarify the mechanism [26]. A previous study has reported that the Dnm3os locus is regulated by TWIST1, which promotes cancer stemness, inflammation, and proliferation of human ovarian cancer cells [30]. In this case, upregulation of $m i R-199 a$ induces pro-inflammatory environment via NF- $\mathrm{kB}$ activation, suggesting that different mechanisms exist between ovarian cancer and synovial sarcoma. Although the exact cell-of-origin of synovial sarcoma remains to be clarified, the results indicate important functions of $m i R-214$ in the growth and differentiation of mesenchymal cells. The cooperative effect of miR-214 for SS18-SSX1 in the mouse model and the association between a high expression of $m i R-214$ with a worse prognosis in human synovial sarcoma cases strongly suggest a role of $m i R-214$ in the aggressive phenotype (Figure 6).

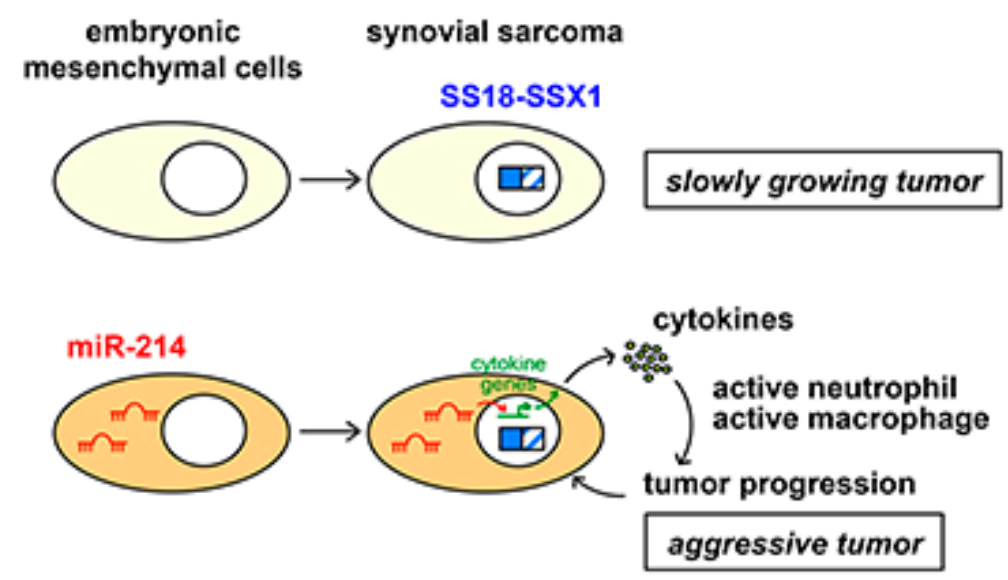

Figure 6. Proposed model of cooperation between SS18-SSX1 and miR-214. SS18-SSX1 expression in embryonic mesenchymal cells that do not express $m i R-214$ induces slowly growing tumors (top). On the other hand, miR-214 upregulates expression of cytokine genes, such as Cxcl15, that activate neutrophils and macrophages in the tumor microenvironment, resulting in an aggressive nature of synovial sarcoma (bottom).

Hirata et al. have recently identified gene fusions between CTDSP1/2 and DNM3OS in a subset of dedifferentiated liposarcomas [31]. The fusions induce upregulation of both DNM3OS and $m i R-214$, as seen in the murine synovial sarcoma with retroviral integration at the Dnm3os locus. Increased expression of DNM3OS and miR-214 was associated with worse prognosis in dedifferentiated liposarcoma, indicating common functions for $m i R-214$ in immature and aggressive phenotypes of soft part sarcomas. 
Despite upregulation of miR-214 in both mouse and human synovial sarcoma [16], exogenous introduction of miR-214 into synovial sarcoma cells did not promote growth, cell migration, or invasion. Tumor suppressor Pten has been reported to be a target of miR-214 in human cancer [32], and its expression was indeed reduced by miR-214. In our study, downregulation of Pten did not show a remarkable growth promoting effect (Figure S5A,B), although a previous study showed that Pten knockout in the SS18-SSX1- and SS18-SSX2-induced synovial sarcoma mouse models promoted invasion and metastasis [33].

miR-214 overexpression in synovial sarcoma cells did not promote cell growth, migration, nor invasion abilities in vitro, suggesting that it might promote tumor development in a cell non-autonomous manner [34,35]. Gene expression profiling revealed upregulation of inflammatory cytokine genes in synovial sarcoma with miR-214 overexpression. Among upregulated cytokine genes Cxcl15 which encodes for IL-8, the mouse homolog of human CXCL8/IL-8, was upregulated both in mouse and human miR-214-overexpressing synovial sarcoma. IL-8 induces migration and activation of granulocytes and macrophages [36,37]. Although miR-214-positive sarcoma failed to show a significant increase in granulocyte or macrophage infiltration in the tumor tissue, increased cytokines might activate intracellular signaling of inflammatory cells to secrete tumor growth promoting factors. Given the high invasive potency of synovial sarcoma cells towards the surrounding tissue, it is possible that IL-8 contributes to the invasive activity of the tumor cells by interacting with the tumor microenvironment. Indeed, increased secretion of IL-8 secretions has been reported in various human malignancies, and IL-8 is involved in more aggressive phenotypes [38-41]. Functional modulation of tumor-associated neutrophils by IL-8 has also been described as a poor prognostic factor for malignancies [39,42]. As the tumor promoting effect of Dnm3os via cancer-associated fibroblasts was also demonstrated in esophageal cancer [43], dynamic modulation of the tumor microenvironment might also be achieved by the Dnm3os/miR-214 axis. In our synovial sarcoma model, nude mice lacking a T-cell population were used given that primary tumors have a slow growth in immunocompetent mice. Nevertheless, significant acceleration of sarcoma development was achieved by $m i R-214$ expression, indicating that T-cell function is dispensable for $m i R$-214-induced modulation of the tumor microenvironment.

The mechanisms by which miR-214 upregulates the expression of cytokine genes remain to be clarified. Modulations of the adenosine A2A receptor and the TGF- $\beta$ signaling by miR-214 have been proposed to have potential roles in cytokine secretion and in the inflammatory response [44,45]. A previous study showed that long non-coding RNA NEAT1 transactivates IL-8 by relocating SFPQ to the nuclear paraspeckle [46]. Similarly, miR-214 might be involved in transactivation of cytokine genes. In conclusion, our study indicates the importance of cooperation between SS18-SSX1 and miR-214 expression in the development and malignant phenotypes of synovial sarcoma. Further studies are needed to clarify the mechanistic role of miR-214 upregulation in synovial sarcoma progression.

\section{Materials and Methods}

\subsection{Plasmid Construction}

N-terminal FLAG-tagged SS18-SSX1 was introduced into the pMYs-IRES-GFP vector. Full-length SS18-SSX1 was cloned from a human synovial sarcoma case. To generate pMYs-SS18-SSX1-IRES-miR-214 and pMYs-SS18-SSX1-IRES-miR-199a2, a mouse 200 bp genomic DNA sequence containing miR-214 or miR-199a2 was introduced by replacing it with the GFP sequence, respectively.

\subsection{Generation and Characterization of the Mouse Synovial Sarcoma Model}

Mouse embryonic mesenchymal cells (eMCs) were prepared as previously described [14]. Briefly, 18.5 dpc embryos were obtained from pregnant BALB/c mice and mesenchymal cells were dissociated from limb soft parts. eMCs were transduced with a pMYs-SS18-SSX1 and $1 \times 10^{6}$ cells were subcutaneously transplanted into nude mice within $48 \mathrm{~h}$ after preparation of eMCs. All animal 
experiments were approved by the animal care committee at the Japanese Foundation for Cancer Research under licenses 10-05-9 and 0604-3-13.

\subsection{Human Sarcoma Specimens}

Synovial sarcoma, rhabdomyosarcoma, myxoid liposarcoma, dermatofibrosarcoma protuberans Ewing sarcoma, and solitary fibrosarcoma surgical specimens were obtained from the adult patients at the Cancer Institute Hospital, Tokyo, Japan after they had provided written informed consent. The study was conducted in accordance with ethical guidelines and approved by Institutional Review Board at the Japanese Foundation for Cancer Research under license 2013-1155.

\subsection{Histology and Immunohistochemistry}

Formaldehyde-fixed, paraffin-embedded tumor tissues were stained with hematoxylin and eosin (H\&E) using standard techniques. Immunohistochemistry was performed using primary antibodies in conjunction with the Histofine Simple Stain kit (Nichirei, Tokyo, Japan). The following primary antibodies were used: Anti-FLAG M2 (Sigma, Burlington, MA, USA), anti-AE1/AE3 (DAKO, Santa Clara, CA, USA), anti-BCL2 (Santa Cruz Biotechnology, Dallas, TX, USA), anti-TLE1 (Santa Cruz Biotechnology), and anti-CD163 (Bioss Antibodies, Woburn, MA, USA).

\subsection{Immunoblotting}

Immunoblotting was performed using whole-cell lysates as previously described [47]. The following primary antibodies were used: Anti-FLAG (M2, Sigma), anti- $\alpha$-tubulin (Sigma), anti-Pten (Cell Signaling Technology, Danvers, MA, USA), anti-Timp2 (Cell Signaling Technology), anti-Ezh1 (Santa Cruz), and anti-Gapdh (Hytest, Turku, Finland).

\subsection{Microarray Analysis}

GeneChip analysis was performed as previously described [14]. Comparison between 12 synovial sarcoma samples, four replicates of eSZ cells, and four replicates of normal human tissue mix consisting of skin, bone marrow, ovary, intestine, kidney, muscle, heart, liver, lung, and brain was achieved. Expression data were analyzed using GeneSpring ver. 14.9 (Agilent Technologies, Santa Clara, CA, USA). For pathway enrichment analysis, the GSEA-P 2.0 software was used [48]. Gene ontology analysis was performed using the Ingenuity Pathway Analysis (IPA) ver. 01-16. The microarray data sets are accessible through the NCBI Gene Expression Omnibus (GEO) database (http://www.ncbi.nlm.nih.gov/geo), with accession number GSE141251. Gene expression data of human sarcoma samples were obtained from the GEO database (GSE20196, GES87437, GSE12102, GSE32569, and GSE66533) [49-53].

\subsection{Real-Time Quantitative Polymerase Chain Reaction (RT-qPCR)}

Total RNA extraction, reverse transcription, and RNA quantification were performed according to previously described methods [12]. MicroRNA isolation was performed using the mirVana miRNA isolation kit (Thermo Fisher Scientific, Waltham, MA, USA). Quantification of microRNA was performed using the TaqMan MicroRNA assay kit (Thermo Fisher Scientific). Probes and primers for pri-miR-214, miR-214_3p, miR-214_5p, pri-miR-199a-2, miR-199a-2_3p, miR-199a-2_5p, sno202, and $u 6$ were purchased from Thermo Fisher Scientific. The sequences of the oligonucleotide primers used are shown in Table S5.

\subsection{Cloning of Retroviral Integration Sites}

Retroviral integration sites were identified by the inverse-PCR method previously described [22,23]. Genomic DNA sequences flanking the retrovirus were cloned into the pGEM T-easy plasmid (Promega, Madison, WI, USA), sequenced, and mapped on mouse chromosome. 


\subsection{In Situ Hybridization}

Expression of miR-214 was detected in formaldehyde-fixed, paraffin-embedded sections of mouse synovial sarcoma using a Hsa-miR-214 probe (BioGenex, Fremont, CA, USA) and the Super Sensitive One-Step Polymer-HRP ISH Detection System (BioGenex) according to the manufacturer's instructions. miR-U6 and miR-Scramble probes (BioGenex) were used as a positive and a negative control, respectively.

\subsection{ELISA}

Mouse synovial sarcoma tissue samples were analyzed using the Mouse Interleukin 8 (IL-8) ELISA kit (MyBioSource, San Diego, CA, USA) according to manufacturer's instructions.

\subsection{Statistical Analysis}

All data are expressed as the mean \pm SEM. Continuous distributions were compared with a two-tailed Student $t$-test. Survival analysis was performed using the Kaplan-Meier life table method, and survival between groups was compared with the log-rank test. The estimated hazard ratio (HR) and its two-sided 95\% confidence interval (CI) were indicated. For significant test results in the miR-214 expression and overall survival analysis, the receiver operating characteristics (ROC) curve was generated to further assess the potential for utility as patient selection markers. Correlation between two groups was tested using Pearson's correlation analysis.

\section{Conclusions}

Our ex vivo mouse model demonstrates both morphological and transcriptional characteristics of human synovial sarcoma. Upregulation of $m i R-214$ accelerates development of mouse synovial sarcoma via modulation of cytokine gene expression and tumor microenvironment, cooperating with SS18-SSX1. Given its association with a worse prognosis in human synovial sarcoma cases, miR-214 can be used as a prognostic biomarker.

Supplementary Materials: The following are available online at http://www.mdpi.com/2072-6694/12/2/324/s1. Table S1: Lists of GSEA enrichment scores, Table S2: Genes upregulated and downregulated in mouse synovial sarcoma and eMCs expressing SS18-SSX1, Table S3: Retroviral integration sites in mouse synovial sarcoma, Table S4: Genes upregulated and downregulated in mouse synovial sarcoma with miR-214 expression, Table S5: The list of PCR primers, Figure S1: Retroviral integrations in mouse synovial sarcoma, Figure S2: Upregulation of miR-214 and Dnm3os in synovial sarcoma, Figure S3: Cumulative tumor incidences of synovial sarcoma co-expressing Fgfr1 or Nedd9, Figure S4: Effects of miR-214 expression in synovial sarcoma, Figure S5: The effect of PTEN silencing in synovial sarcoma development.

Author Contributions: M.T. performed the research, analyzed the data, and wrote the paper; M.H. contributed to establish the mouse model; Y.Y. performed the histological and biochemical analyses; K.A. and S.M. analyzed the clinical data; S.S. performed bioinformatic analyses; T.N. designed and supervised the study and wrote the paper. All authors have read and agreed to the published version of the manuscript.

Funding: The study was supported by the Grants-in-Aid for Scientific Research from Japan Society for the Promotion of Science (no. 26250029 to T.N., and no. 19K07702 to M.T.), and by a Grant-in-Aid for Project for Cancer Research and Therapeutic Evolution from Japan Agency for Medical Research and Development (no. 17cmA002 to T.N.).

Acknowledgments: The authors thank Ayano Imakawa for her technical assistance.

Conflicts of Interest: The authors declare no conflict of interest.

\section{References}

1. Enzinger, F.M.; Weiss, S.W. Synovial sarcoma. In Soft Tissue Tumors, 5th ed.; Weiss, S.W., Goldblum, J.R., Eds.; Elsevier: Philadelphia, PA, USA, 2008; pp. 1161-1182.

2. Clark, J.; Rocques, P.J.; Crew, A.J.; Gill, S.; Shipley, J.; Chan, A.M.; Gusterson, B.A.; Cooper, C.S. Identification of novel genes, SYT and SSX, involved in the $t(X ; 18)(p 11.2 ; q 11.2)$ translocation found in human synovial sarcoma. Nat. Genet. 1994, 7, 502-508. [CrossRef] [PubMed] 
3. Kawai, A.; Woodruff, J.; Healey, J.H.; Brennan, M.F.; Antonescu, C.R.; Ladanyi, M. SYT-SSX gene fusion as a determinant of morphology and prognosis in synovial sarcoma. N. Engl. J. Med. 1998, 338, 153-160. [CrossRef] [PubMed]

4. Ladanyi, M. Fusions of the SYT and SSX genes in synovial sarcoma. Oncogene 2001, 20, 5755-5762. [CrossRef]

5. Nielsen, T.O.; Poulin, N.M.; Ladanyi, M. Synovial sarcoma: Recent discoveries as a roadmap to new avenues for therapy. Cancer Discov. 2015, 4, 123-134. [CrossRef]

6. Kadoch, C.; Crabtree, G.G. Reversible disruption of mSWI/SNF (BAF) complexes by the SS18-SSX oncogenic fusion in synovial sarcoma. Cell 2013, 153, 71-85. [CrossRef]

7. McBride, M.J.; Pulice, J.L.; Beird, H.C.; Ingram, D.R.; D'Avino, A.R.; Shern, J.F.; Charville, G.W.; Hornick, J.L.; Nakayama, R.T.; Garcia-Rivera, E.M.; et al. The SS18-SSX fusion oncoprotein hijacks BAF complex targeting and function to drive synovial sarcoma. Cancer Cell 2018, 33, 1128-1141. [CrossRef]

8. Su, L.; Sampaio, A.V.; Jones, K.B.; Pacheco, M.; Goytain, A.; Lin, S.; Poulin, N.; Yi, L.; Rossi, F.M.; Kast, J.; et al. Deconstruction of the SS18-SSX fusion oncoprotein complex: Insights into disease etiology and therapeutics. Cancer Cell 2012, 21, 333-347. [CrossRef]

9. Barham, W.; Frump, A.L.; Sherill, T.P.; Garcia, C.B.; Saito-Diaz, K.; VanSaun, M.N.; Fingleton, B.; Gleaves, L.; Orton, D.; Capecchi, M.R.; et al. Targeting the Wnt pathway in synovial sarcoma models. Cancer Discov. 2013, 3, 1286-1301. [CrossRef]

10. Banito, A.; Li, X.; Laporte, A.N.; Roe, J.S.; Sanchez-Vega, F.; Huang, C.H.; Dancsok, A.R.; Hatzi, K.; Chen, C.C.; Tschaharganeh, D.F.; et al. The SS18-SSX oncoprotein hijacks KDM2B-PRC1.1 to drive synovial sarcoma. Cancer Cell 2018, 33, 527-541. [CrossRef] [PubMed]

11. Vlenterie, M.; Hillebrandt-Roeffen, M.H.S.; Flucke, U.E.; Gronen, P.J.T.A.; Tops, B.B.J.; Kamping, E.J.; Pfundt, R.; de Bruijn, D.R.H.; van Kessel, A.H.M.G.; van Krieken, H.J.H.J.M.; et al. Next generation sequencing in synovial sarcoma reveals novel gene mutations. Oncotarget 2015, 6, 34680-34690. [CrossRef]

12. Haldar, M.; Hancock, J.D.; Coffin, C.M.; Lessnick, S.L.; Capecchi, M.R. A conditional mouse model of synovial sarcoma: Insights into a myogenic origin. Cancer Cell 2007, 11, 375-388. [CrossRef]

13. Tanaka, M.; Yamazaki, Y.; Kanno, Y.; Igarashi, K.; Aisaki, K.; Kanno, J.; Nakamura, T. Ewing's sarcoma precursors are highly enriched in embryonic osteochondrogenic progenitors. J. Clin. Investig. 2014, 121, 3061-3074. [CrossRef]

14. Tanaka, M.; Homme, M.; Yamazaki, Y.; Shimizu, R.; Takazawa, Y.; Nakamura, T. Modeling alveolar soft part sarcoma unveils novel mechanisms of metastasis. Cancer Res. 2017, 77, 897-904. [CrossRef] [PubMed]

15. Yoshimoto, T.; Tanaka, M.; Homme, M.; Yamazaki, Y.; Takazawa, Y.; Antonescu, C.R.; Nakamura, T. CIC-DUX4 induces small round cell sarcoma distinct from Ewing sarcoma. Cancer Res. 2017, 77, 2927-2937. [CrossRef] [PubMed]

16. Subramanian, S.; Lui, W.O.; Lee, C.H.; Espinosa, I.; Nielsen, T.O.; Heinrich, M.C.; Corless, C.L.; Fire, A.Z.; van de Rijn, M. MicroRNA expression signature of human sarcomas. Oncogene 2008, 27, 2015-2026. [CrossRef]

17. Terry, J.; Saito, T.; Subramanian, S.; Ruttan, C.; Antonescu, C.R.; Goldblum, J.R.; Downs-Kelly, E.; Corless, C.L.; Rubin, B.P.; van de Rijn, M.; et al. TLE1 as a diagnostic immunohistochemical marker for synovial sarcoma emerging from gene expression profiling studies. Am. J. Surg. Pathol. 2007, 31, 240-246. [CrossRef] [PubMed]

18. Krskova, L.; Kalinova, M.; Brizova, H.; Mrhalova, M.; Sumerauer, D.; Kodet, R. Molecular and immunohistochemical analyses of BCL2, KI-67, and cyclin D1 expression in synovial sarcoma. Cancer Genet. Cytogenet. 2009, 193, 1-8. [CrossRef]

19. Isakoff, M.S.; Sansam, C.G.; Tamayo, P.; Subramanian, A.; Evans, J.A.; Fillmore, C.M.; Wang, X.; Biegel, J.A.; Pomeroy, S.L.; Mesirov, J.P.; et al. Inactivation of the Snf5 tumor suppressor stimulates cell cycle progression and cooperates with p53 loss in oncogenic transformation. Proc. Natl. Acad. Sci. USA 2005, 102, 17445-17450. [CrossRef] [PubMed]

20. Jones, K.B.; Su, L.; Jin, H.; Lenz, C.; Randall, R.L.; Underhill, T.M.; Nielsen, T.O.; Sharma, S.; Capecchi, M.R. SS18-SSX2 and the mitochondrial apoptosis pathway in mouse and human synovial sarcoma. Oncogene 2013, 32, 2365-2371. [CrossRef]

21. Endo, M.; Su, L.; Nielsen, T.O. Activating transcription factor 2 in mesenchymal tumors. Hum. Pathol. 2014, 45, 276-284. [CrossRef]

22. Lanzi, C.; Dal Bo, L.; Favini, E.; Tortoreto, M.; Beretta, G.L.; Arrighetti, N.; Zaffaroni, N.; Cassinelli, C. Overactive IGF1/insulin receptors and NRASQ61R mutation drive mechanisms of resistance to Pazopanib and define rational combination strategies to treat synovial sarcoma. Cancers 2019, 11, E408. [CrossRef] 
23. Nakamura, T. Retroviral insertional mutagenesis identifies oncogene cooperation. Cancer Sci. 2005, 96, 7-12. [CrossRef]

24. Jin, G.; Yamazaki, Y.; Takuwa, M.; Takahara, T.; Kaneko, K.; Kuwata, T.; Miyata, S.; Nakamura, T. Trib1 and Evi1 cooperate with Hoxa and Meis1 in myeloid leukemogenesis. Blood 2007, 109, 3998-4005. [CrossRef] [PubMed]

25. Tanaka, M.; Jin, G.; Yamazaki, Y.; Takahara, T.; Takuwa, M.; Nakamura, T. Identification of candidate cooperative genes of the Apc mutation in transformation of the colon epithelial cell by retroviral insertional mutagenesis. Cancer Sci. 2008, 99, 979-985. [CrossRef] [PubMed]

26. Watanabe, T.; Sato, T.; Amano, T.; Kawamura, Y.; Kawamura, N.; Kawaguchi, H.; Yamashita, N.; Kurihara, H.; Nakaoka, T. Dnm3os, a non-coding RNA, is required for normal growth and skeletal development in mice. Dev. Dyn. 2008, 237, 3738-3748.

27. Desvignes, T.; Contreras, A.; Postlethwair, J.H. Evolution of the miR199-214 cluster and vertebrate skeletal development. RNA Biol. 2014, 11, 281-294. [CrossRef]

28. Loebel, D.A.; Tsoi, B.; Wong, N.; Tam, P.P. A conserved noncoding intronic transcript at the mouse Dnm3 locus. Genomics 2005, 85, 782-789. [CrossRef]

29. Roberto, V.P.; Gavaia, P.; Nunes, M.J.; Rodrigues, E.; Cancela, M.L.; Tiago, D.M. Evidences for a new role of miR-214 in chondrogenesis. Sci. Rep. 2018, 8, 3704. [CrossRef]

30. Yin, G.; Chen, R.; Alvero, A.B.; Fu, H.H.; Holmberg, J.; Glackin, C.; Rutherford, T.; Mor, G. TWISTing stemness, inflammation and proliferation of epithelial ovarian cancer cells through MIR199A2/214. Oncogene 2010, 29, 3545-3553. [CrossRef] [PubMed]

31. Hirata, M.; Asano, N.; Katayama, K.; Yoshida, A.; Tsuda, Y.; Sekimizu, M.; Mitani, S.; Kobayashi, E.; Komiyama, M.; Fujimoto, H.; et al. Integrated exome and RNA sequencing of dedifferentiated liposarcoma. Nat. Commun. 2020, in press. [CrossRef]

32. Yang, H.; Kong, W.; He, L.; Zhao, J.J.; O’Donell, J.D.; Wang, J.; Wenham, R.M.; Coppola, D.; Kruk, P.A.; Nicosia, S.V.; et al. MicroRNA expression profiling in human ovarian cancer: miR-214 induces cell survival and cisplatin resistance by targeting PTEN. Cancer Res. 2008, 68, 425-433. [CrossRef] [PubMed]

33. Barrott, J.J.; Kafchinski, L.A.; Jin, H.; Potter, J.W.; Kannan, S.D.; Kennedy, R.; Mosbruger, T.; Wang, W.L.; Tsai, J.W.; Araujo, D.M.; et al. Modeling synovial sarcoma metastasis in the mouse: PI3'-lipid signaling and inflammation. J. Exp. Med. 2016, 213, 2989-3005. [CrossRef] [PubMed]

34. Fang, H.Y.; Greten, F.R. Cell autonomous and non-autonomous functions of IKK and NF- B during the pathogenesis of gastrointestinal tumors. Cancers 2011, 3, 2214-2222. [CrossRef]

35. Bellazzo, A.; Di Minin, G.; Valentino, E.; Sicari, D.; Torre, D.; Marchionni, L.; Serpi, F.; Stadler, M.B.; Taverna, D.; Zuccolotto, G.; et al. Cell-autonomous and cell non-autonomous downregulation of tumor suppressor DAB2IP by microRNA-149-3p promotes aggressiveness of cancer cells. Cell Death Differ. 2018, 25, 1224-1238. [CrossRef]

36. Baggiolini, M.; Clark-Lewis, I. Interleukin-8, a chemotactic and inflammatory cytokine. FEBS Lett. 1992, 307, 97-101. [CrossRef]

37. Xie, K. Interleukin-8 and human cancer biology. Cytokine Growth Factor Rev. 2001, 12, 375-391. [CrossRef]

38. Abdul-Aziz, A.M.; Shafat, M.S.; Mehta, T.K.; Di Palma, F.; Lawes, M.J.; Rushworth, S.A.; Bowles, K.M. MIF-induced stromal PKC/IL8 is essential in human acute myeloid leukemia. Cancer Res. 2017, 77, 303-311. [CrossRef] [PubMed]

39. Manfroi, B.; McKee, T.; Mayol, J.F.; Tabruyn, S.; Moret, S.; Villiers, C.; Righini, C.; Dyer, M.; Callanan, M.; Schneider, P.; et al. CXCL-8/IL8 produced by diffuse large B-cell lymphomas recruits neutrophils expressing a proliferation-inducing ligand APRIL. Cancer Res. 2017, 77, 1097-1107. [CrossRef] [PubMed]

40. Kahraman, D.C.; Kahraman, T.; Cetin-Atalay, R. Targeting PI3K/Akt/mTOR pathway identifies differential expression and functional role of IL8 in liver cancer stem cell enrichment. Mol. Cancer Ther. 2019, 18, $2146-2157$. [PubMed]

41. Maynard, J.P.; Ertunc, O.; Kulac, I.; Baena-Del Valle, J.A.; De Marzo, A.M.; Sfanos, K.S. IL8 expression is associated with prostate cancer aggressiveness and androgen receptor loss in primary and metastatic prostate cancer. Mol. Cancer Res. 2019. [CrossRef]

42. Nie, M.; Yang, L.; Bi, X.; Wang, Y.; Sun, P.; Yang, H.; Liu, P.; Li, Z.; Xia, Y.; Jiang, W. Neutrophil extracellular traps induced by IL8 promote diffuse large B-cell lymphoma progression via the TLR9 signaling. Clin. Cancer Res. 2019, 25, 1867-1879. [CrossRef] [PubMed] 
43. Zhang, H.; Hua, Y.; Jiang, Z.; Yue, J.; Shi, M.; Zhen, X.; Zhang, X.; Yang, L.; Zhou, R.; Wu, S. Cancer-associated fibroblast-promoted lnc RNA DNM3OS confers radioresistance by regulating DNA damage response in esophageal squamous cell carcinoma. Clin. Cancer Res. 2019, 25, 1989-2000. [CrossRef]

44. Zhao, L.; Liu, Y.W.; Yang, T.; Gan, L.; Yamg, N.; Dai, S.S.; He, F. The mutual regulation between miR-214 and A2AR signaling plays an important role in inflammatory response. Cell Signal. 2015, 27, 2026-2034. [CrossRef]

45. Kuninty, P.R.; Bojmar, L.; Tjomsland, V.; Larsson, M.; Storm, G.; Ostman, A.; Sandstrom, P.; Prakash, J. MicroRNA-199a and -214 as potential therapeutic targets in pancreatic stellate cells in pancreatic tumor. Oncotarget 2016, 7, 16396-16408. [CrossRef]

46. Imamura, K.; Imamachi, N.; Akizuki, G.; Mumakura, M.; Kawaguchi, A.; Nagata, K.; Kato, A.; Kawaguchi, Y.; Sato, H.; Yoneda, M.; et al. Long noncoding RNA NEAT1-dependent SFPQ relocation from promoter region to paraspeckle mediates IL8 expression upon immune stimuli. Mol. Cell 2014, 53, 393-406. [CrossRef]

47. Kawamura-Saito, M.; Yamazaki, Y.; Kaneko, K.; Kawaguchi, N.; Kanda, H.; Mukai, H.; Gotoh, T.; Motoi, T.; Fukayama, M.; Takizawa, T.; et al. Fusion between CIC and DUX4 up-regulates PEA3 family genes in Ewing-like sarcomas with $\mathrm{t}(4 ; 19)(\mathrm{q} 35 ; \mathrm{q} 13)$ translocation. Hum. Mol. Genet. 2006, 15, 2125-2137. [CrossRef]

48. Subramanian, A.; Kuehn, H.; Gould, J.; Tamayo, P.; Mesirov, J.P. GSEA-P: A desktop application for gene set enrichment analysis. Bioinformatics 2007, 23, 3251-3253. [CrossRef] [PubMed]

49. Nakayama, R.; Mitani, S.; Nakagawa, T.; Hasegawa, T.; Kawai, A.; Morioka, H.; Yabe, H.; Toyama, Y.; Ogose, A.; Toguchida, J.; et al. Gene expression profiling of synovial sarcoma: Distinct signature of poorly differentiated type. Am. J. Surg. Pathol. 2010, 34, 1599-1607. [CrossRef]

50. Vella, S.; Tavanti, E.; Hattinger, C.M.; Fanelli, M.; Versteeg, R.; Koster, J.; Picci, P.; Serra, M. Targeting CDKs with Roscovitine increases sensitivity to DNA damaging drugs of human osteosarcoma cells. PLoS ONE 2016, 11, e0166233. [CrossRef] [PubMed]

51. Scotlandi, K.; Remondini, D.; Castellani, G.; Manara, M.C.; Nardi, F.; Cantiani, L.; Francesconi, M.; Mercuri, M.; Caccuri, A.M.; Serra, M.; et al. Overcoming resistance to concventional drugs in Ewing sarcoma and identification of molecular predictors of outcome. J. Clin. Oncol. 2009, 27, 2209-2216. [CrossRef]

52. Kummar, S.; Allen, D.; Monks, A.; Polley, E.C.; Hose, C.D.; Ivy, S.P.; Turkbey, I.B.; Lawrence, S.; Kinders, R.J.; Choyke, P.; et al. Cediranib for metastatic alveolar soft part sarcoma. J. Clin. Oncol. 2013, 31, 2296-2302. [CrossRef] [PubMed]

53. Sun, W.; Chatterjee, B.; Wang, Y.; Stevenson, H.S.; Edelman, D.C.; Meltzer, P.S.; Barr, F.G. Distinct methylation profiles characterize fusion-positive and fusion-negative rhabdomyosarcoma. Mod. Pathol. 2015, 28, 1214-1224. [CrossRef] [PubMed]

(C) 2020 by the authors. Licensee MDPI, Basel, Switzerland. This article is an open access article distributed under the terms and conditions of the Creative Commons Attribution (CC BY) license (http://creativecommons.org/licenses/by/4.0/). 